\title{
ESTUDO DA INCIDÊNCIA DE INFECÇÃO ATIVA POR CITOMEGALOVÍRUS EM TRANSPLANTE PULMONAR E PROFILAXIA UNIVERSAL COM GANCICLOVIR INTRAVENOSO
}

\author{
The Incidence of Cytomegalovirus Infection in Lung Transplant Recipients under Universal \\ Prophylaxis with Intravenous Ganciclovir
}

\begin{abstract}
Regina Barbosa Schröeder', Tatiana Michelon², João Wurdig', Iara Fagundes ${ }^{4}$, Sadi Schio ${ }^{5}$, Leticia Sanchez ${ }^{5}$, Teresa Sukienik ${ }^{6}$, Jose Camargo ${ }^{7}$, Alessandro Pasqualotto ${ }^{8}$, Jorge Neumann ${ }^{9}$
\end{abstract}

\begin{abstract}
RESUMO
Objetivo: determinar a incidência de infecção ativa por citomegalovírus em transplante pulmonar sob profilaxia universal com ganciclovir intravenoso e analisar a sua repercussão na sobrevida após o transplante. Pacientes e Métodos: Foram estudados 82 entre os 106 transplantes realizados entre Mar/99 e Fev/04, excluindo-se os óbitos no lo mês ( $n=24 ; 22,6 \%)$. Todos receberam ciclosporina, azatioprina e prednisona. A dose de ganciclovir profilático foi de $10 \mathrm{mg} / \mathrm{kg} / \mathrm{dia}$ nas 3 primeiras semanas e $5 \mathrm{mg} / \mathrm{kg} / \mathrm{dia}, 3 \mathrm{x} / \mathrm{sem}$ da $4 \mathrm{a}$ à 12 a semanas. Na vigência de infecção ativa, tratou-se com ganciclovir $10 \mathrm{mg} / \mathrm{kg} /$ dia até negativar a antigenemia ou completar 21 dias. A monitorização da infecção foi com imunoistoquímica para o antígeno pp65 do citomegalovírus em sangue periférico, semanal entre a 3a e $12^{\underline{a}}$ semanas e mensal entre 40 e 120 meses ou quando suspeita clínica. Definiu-se como infecção ativa qualquer positividade da antigenemia em 105 granulócitos. Empregaram-se t Student, Qui-quadrado, Exato de Fisher, média e desvio-padrão, Kaplan Meier, sendo p<0,05. Resultados: A incidência de infecção foi de $68,3 \%$ (56/82). A mediana da antigenemia máxima foi de 11 células (1-1096). A mediana de tempo para positivar a antigenemia foi de 114 dias (28-343). Não houve diferença em relação ao gênero $(p=0,804)$, idade $(p=0,599)$ e tipo de doador $(p=0,375)$ entre os grupos com e sem infecção. A taxa de óbito em 5 anos foi de 40,2\% (33/82), sendo 19,5\% (16/82) no primeiro ano, sem diferença entre os grupos. Conclusão: A incidência de infecção ativa por citomegalovírus foi elevada a despeito do regime profilático monoterápico com ganciclovir intravenoso.
\end{abstract}

Descritores: citomegalovírus, antigenemia, transplante pulmonar, ganciclovir.

Trabalho realizado no Laboratório de Imunologia de Transplantes da Santa Casa de Porto Alegre.

1. Bióloga do Laboratório de Imunologia de Transplantes da Santa Casa de Porto Alegre, Mestre em Ciências da Saúde.

2. Médica do Laboratório de Imunologia de Transplantes da Santa Casa de Porto Alegre, doutora em Patologia Pesquisadora PRODOC-CAPES do programa de Pós-Graduação em Patologia da Fundação Faculdade Federal de Ciências Médicas de Porto AlegreFFFCMPA.

3. Estudante de Biologia e Funcionário do Laboratório de Imunologia de Transplantes da Santa Casa de Porto Alegre.

4. Farmacêutica do Laboratório de Imunologia de Transplantes da Santa Casa de Porto Alegre.

5. Médico do Serviço de Transplante Pulmonar da Santa Casa de Porto Alegre.

6. Médico Responsável pelo Serviço de Controle de Infecção Hospitalar da Santa Casa de Porto Alegre.

7. Médico Responsável pelo Serviço de Transplante Pulmonar da Santa Casa de Porto Alegre.

8. Médico do Serviço de Controle de Infecção Hospitalar da Santa Casa de Porto Alegre.

9. Médico Diretor do Laboratório de Imunologia de Transplantes da Santa Casa de Porto Alegre.

Endereço para correspondência: Regina Barbosa Schröeder - Hospital Dom Vicente Scherer, Av Independência 75 - Bairro Centro - CEP 94035-075 - Porto Alegre - RS - Brasil Telefone/Fax: (51) $32148670 / 32148629$ E-mail: regina@santacasa.tche.br

Recebido em: 19/06/05 Aceito em: 05/07/05

\section{INTRODUÇÃO}

Citomegalovírus (CMV) é o agente infeccioso mais freqüente entre os pacientes imunodeprimidos e/ou imunossuprimidos, com manifestações clínicas muito variáveis. Está associado a aumento da imunogenicidade do órgão transplantado com conseqüente aumento do risco de rejeição ao enxerto e de infecções oportunistas. ${ }^{1,2,3,4}$

A incidência de morbidade secundária a esta infecção é elevada após o transplante dos diferentes órgãos e também de medula óssea. Em transplante pulmonar, este risco é especialmente importante uma vez que uma das manifestações clínicas é a pneumonite por CMV, a qual pode ser confundida com processos de rejeição aguda ou processos neoplásicos e a sua ocorrência costuma ser concomitante ao período de risco inicial pós-transplante (primeiros 3 meses). ${ }^{5} \mathrm{O}$ surgimento de viremia pode estar associado ao desenvolvimento de bronquiolite obliterante e, em transplante pulmonar pediátrico, está associada a maior risco de morte e de retransplante. ${ }^{6,7}$

Com a disponibilidade da utilização terapêutica do ganciclovir, muitos centros de transplante passaram a utilizar esta mesma droga em diferentes esquemas de profilaxia, sem haver, no entanto, estudos clínicos randomizados para esta avaliação. Concomitantemente, os 
métodos diagnósticos específicos e altamente sensíveis passaram a ser disponibilizados na maioria dos centros, o que torna ainda mais discutível a utilização da única droga eficaz na terapia, com alto custo e toxicidade, como a droga de escolha também para a profilaxia desta infecção que é altamente prevalente. ${ }^{8,9,10}$

Diante da experiência com a utilização da terapia preemptiva baseada na avaliação sistemática da antigenemia em outros transplantes, propomo-nos a avaliar o período de 5 anos de prática de profilaxia universal com ganciclovir intravenoso em transplante pulmonar, considerando a incidência de infecção ativa e a sua repercussão na sobrevida destes pacientes.

\section{Pacientes e métodos}

Entre o período de Março/94 e Fevereiro/04 foram realizados 106 transplantes pulmonares na instituição. Para propósitos deste estudo, foram excluídos 24 pacientes devido à ocorrência de óbito no primeiro mês e não realização sistemática da pesquisa de infecção ativa por CMV neste período inicial. Fizeram parte do estudo, 82 pacientes, todos submetidos a um esquema tríplice de imunossupressão que incluía ciclosporina, azatioprina e prednisona.

Os receptores, independente da sorologia inicial do par doadorreceptor, foram submetidos a profilaxia com ganciclovir intravenoso (GIV) em doses de $10 \mathrm{mg} / \mathrm{kg} /$ dia durante as 3 primeiras semanas pós-transplante e, após, dose de $5 \mathrm{mg} / \mathrm{kg} /$ dia entre a $4^{\mathrm{a}}$ e a $12^{\mathrm{a}}$ semanas. Os pacientes foram sistematicamente avaliados pela pesquisa semanal de antigenemia para o antígeno pp65 do CMV entre a $3^{\mathrm{a}}$ e a $12^{\mathrm{a}}$ semanas pós-transplante, período de maior risco para o desenvolvimento de doença pelo CMV. Entre 40 e 120 meses pós-transplante, a antigenemia foi coletada quinzenalmente e, após, sempre que houvesse suspeita clínica.

Pacientes com sinais ou sintomas clínicos sugestivos de doença por CMV e evidência de viremia detectada pela antigenemia foram tratados com GIV em dose de $10 \mathrm{mg} / \mathrm{kg} /$ dia até negativar a antigenemia, avaliada semanalmente neste período, ou completar 21 dias de tratamento.

A pesquisa de antigenemia foi realizada através de imunoistoquímica com anticorpo monoclonal para o antígeno pp65 do CMV (ClonabBiotest, Germany) e seu resultado expresso em número de células positivas em 105 granulócitos isolados de sangue periférico colhido com EDTA. Viremia foi determinada pela presença de pelo menos 1 células positiva/105 granulócitos circulantes.

As respectivas incidências dos desfechos analisados foram descritas através de percentagem. A análise estatística empregou teste $t$ de Student, qui-quadrado, Exato de Fisher, média e desvio-padrão e método de Kaplan-Meier para a estimativa da sobrevida. O intervalo de confiança adotado foi de $95 \%$ e os valores de $\mathrm{P}<0,05$ foram considerados significativos.

\section{RESULTADOS}

Os 24 pacientes com óbito no primeiro mês foram excluídos deste estudo e representaram uma incidência de mortalidade de $22,6 \%$ neste período. Desta forma, a sobrevida global ao final de 30 dias de transplante foi de $77,3 \%$ e estes pacientes constituíram a amostra estudada para o desfecho de infecção por CMV.

A incidência de infecção ativa por CMV no primeiro ano póstransplante entre os receptores de transplante pulmonar sob profilaxia com GIV foi de 68,3\% (56/82). Entre eles, 46,4\% (26/56) tiveram até 10 células positivas na antigenemia, enquanto os demais $(53,6 \% ; 30 / 56)$ tiveram uma positividade superior, variando de 11 a 1096 células. Desta forma, 36,6\% (30/82) da amostra apresentou antigenemia positiva acima de 10 células a despeito da profilaxia universal com GIV. No estudo da antigenemia entre os pacientes que desenvolveram viremia no primeiro ano $(n=56)$, observou-se uma variação de 1 a 1096 células positivas, com mediana de 11 células $(59,6+157,9)$. O tempo necessário para o desenvolvimento de antigenemia positiva variou de 28 a 343 dias pós-transplante, com mediana de 114 dias (3,8 meses).

A descrição das respectivas incidências de infecção ativa por CMV está descrita na Figura 1. As características demográficas dos pacientes que desenvolveram ou não a infecção ativa por CMV no primeiro ano pós-transplante foram semelhantes e podem ser observadas na Tabela 1 .

Tabela 1. Características demográficas dos receptores de transplante pulmonar conforme o desenvolvimento ou não de infecção ativa por Citomegalovírus no primeiro ano póstransplante

\begin{tabular}{lccc}
\hline & Sem Viremia $n=26$ & Sem Viremia $n=26$ & P / RR \\
\hline Gênero $(\mathrm{n} ; \%)$ & $18(69,2)$ & $36(64,2)$ & 0,80 \\
Doador Cadáver(n;\%) & $23(88,5)$ & $53(94,6)$ & 0,37 \\
Idade (média \pm DP) & $49,9 \pm 17,4$ & & 0,59 \\
& & $51,9 \pm 14,9$ & 9 \\
\hline
\end{tabular}

Houve suspeita clínica de infecção ativa por CMV após o primeiro ano de transplante em 59 pacientes que foram submetidos a pesquisa de antigenemia tardiamente. Entre aqueles pacientes que permaneceram sempre negativos no acompanhamento do primeiro ano, 31\% (4/13) manifestaram antigenemia positiva no segundo ano. Entre os pacientes que fizeram viremia no primeiro ano, $50 \%$ (23/46) reativou a infecção por CMV tardiamente $(\mathrm{P}=0,345)$.

Na comparação entre os grupos positivo e negativo para infecção ativa por CMV no primeiro ano, não houve diferença quanto à taxa de óbito geral no período de estudo, óbito no primeiro ano e óbito após o primeiro ano de transplante. A incidência de óbito no período de estudo foi de $37,6 \%$ (21/51) no grupo positivo e de $46,2 \%$ $(12 / 26)$ no grupo negativo $(\mathrm{P}=0,478 ; \mathrm{RR}=1,1 ; \mathrm{IC}: 0,8-1,5)$. Óbito no primeiro ano ocorreu em $12,5 \%(7 / 56)$ dos pacientes com viremia no primeiro ano e em $34,6 \%(9 / 26)$ dos pacientes que permaneceram negativos $(\mathrm{P}=0,033 ; \mathrm{RR}=1,7 ; \mathrm{IC}: 1,0-3,0)$. A taxa de óbito após o primeiro ano foi de $28,6 \%$ entre os pacientes positivos e de $17,6 \%$ entre os negativos ( $\mathrm{P}=0,525 ; \mathrm{RR}=1,1 ; \mathrm{IC}: 0,9-1,5)$.

$\mathrm{Na}$ análise da sobrevida atuarial, observamos uma taxa de sobrevida no final do primeiro ano de $80,5 \%$ entre os pacientes da amostra estudada, significando uma sobrevida global de $62,2 \%$ se considerarmos os pacientes com óbito no primeiro mês. A curva de sobrevida foi semelhante entre os pacientes com e sem viremia no primeiro ano $(\mathrm{P}=0,204)$ e também não diferiu entre os pacientes que fizeram antigenemia até 10 células ou mais do que 10 células positivas em 105 granulócitos $(\mathrm{P}=0,128)$. As respectivas curvas de sobrevida podem ser observadas nas Figuras 1 e 2 . 


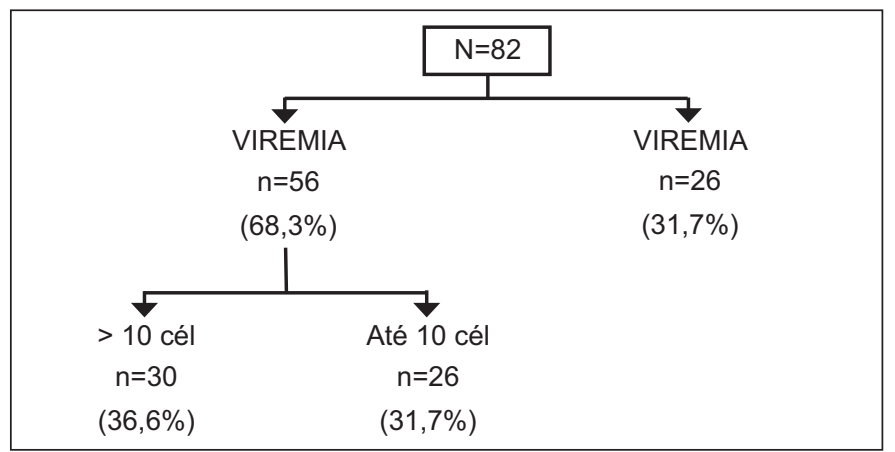

Figura 1. Epidemiologia da infecção por citomegalovírus no primeiro ano pós-transplante pulmonar no período de Março/99 e Fevereiro/04 sob profilaxia universal com Ganciclovir Intravenoso.

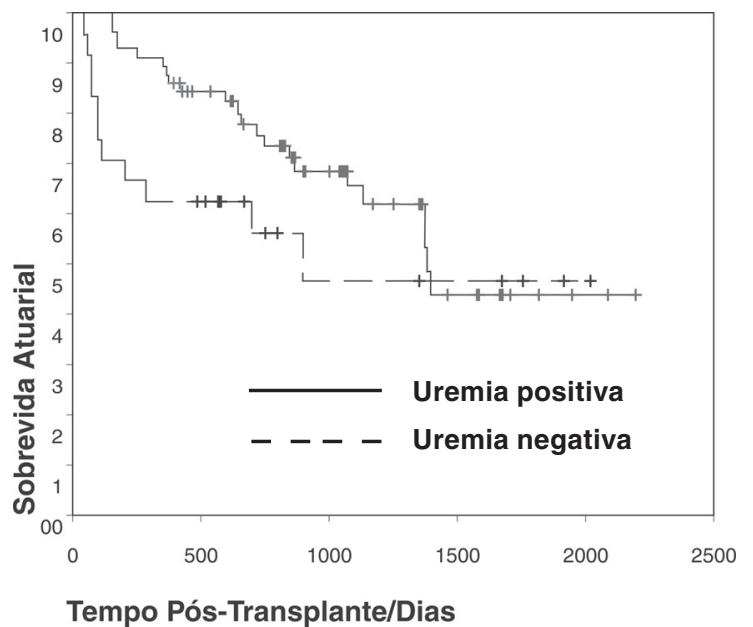

Figura 2. Sobrevida do transplante pulmonar entre Março/99 e Fevereiro/04 comparando pacientes com e sem viremia para citomegalovírus no primeiro ano pós-transplante

\section{DISCUSSÃO}

A maioria dos estudos relata incidência de viremia de $30 \%$ a $90 \%$ em pacientes submetidos a transplantes, com variação de $20 \%$ a $60 \%$ de incidência de doença por CMV. ${ }^{5,11}$ Neste estudo, observouse incidência de $68 \%$ de viremia entre os receptores de transplante pulmonar, a despeito da profilaxia universal realizada com GIV.

Em nossa instituição a experiência com a terapia preemptiva em transplante renal revela uma incidência muito semelhante, com taxas de $60 \%$ de viremia e de $38 \%$ de doença por CMV com necessidade de terapia plena com GIV nos primeiros 3 meses. ${ }^{12}$ Considerando-se que o ganciclovir constitui a única droga disponível para uso clínico nos dias de hoje, esta droga tem sido empregada tanto por via oral como por via intravenosa em ambos os contextos clínicos - profilaxia e/ou tratamento de doença viral ativa. Alternativas nem sempre disponíveis e igualmente com alto custo são a globulina hiperimune específica anti-CMV e/ou o valganciclovir, ambas ainda sem comprovação de superioridade isolada ou em associação com o ganciclovir. .1,14,15 $^{13}$

Não existe uniformidade de conduta, de modo que os diferentes centros adotam diferentes protocolos de profilaxia e/ou tratamento com ganciclovir e sua combinação com outras drogas o que dificulta a comparação entre eles. ${ }^{15,16,17,18}$ A profilaxia pode ser realizada apenas para pacientes com alto risco de desenvolvimento de doença ativa (ex: receptor IgG negativo para CMV) ou de forma universal levando-se em conta a alta incidência da doença em qualquer tipo de transplante. A realização de tratamento clínico, com doses plenas de ganciclovir, também pode ser administrado somente a pacientes com sintomatologia importante ou, de outra forma, preemptiva, contemplando os casos de detecção precoce da replicação viral.

O propósito deste estudo foi avaliar o benefício da utilização da terapia profilática universal com GIV no transplante pulmonar realizada durante um período de 5 anos em nossa instituição. A grande vantagem almejada com esta conduta seria a observação de uma baixa incidência de infecção ativa por CMV, o que contrasta com a taxa de $68 \%$ observada. Outra potencial vantagem seria a baixa incidência de doença grave por CMV, e isto pode ser depreendido a partir da celularidade observada no estudo (1/3 dos pacientes com $>10$ células positivas e mediana de 11 células entre os pacientes que desenvolveram viremia no primeiro ano). Alguns autores demonstram claramente que a profilaxia com ganciclovir oral e/ou intravenoso é capaz de evitar casos de doença grave e de reduzir a incidência dos episódios de rejeição. Todavia não permite a redução da incidência ou da progressão de bronquiolite obliterante ou sobrevida do transplante pulmonar. ${ }^{19}$

Apesar de não ter sido abordado neste estudo, as desvantagens da profilaxia universal com GIV incluem o alto custo da droga e o seu potencial de toxicidade e de indução de resistência viral à única medicação disponível para uso clínico, tanto para profilaxia como para tratamento de doença ativa. A primeira descrição de resistência do CMV ao ganciclovir foi em 1986 e vem sendo observada cada vez mais entre os receptores de transplantes de órgãos sólidos. Em transplante de pulmão, pode ser observada em até $10 \%$ dos casos, sendo mais freqüente quanto maior o número de reativações virais ao longo do tempo e principalmente em receptores IgG negativos. 9,20 O grande problema é que, com a utilização do ganciclovir em larga escala, estamos certamente assumindo o risco de desenvolver resistência à única droga disponível e nem sequer dispomos de tecnologia diagnóstica para a detecção de eventual resistência viral em nosso meio.

A pesquisa de antigenemia para o antígeno pp 65 do CMV constitui um método diagnóstico sensível, específico e precoce para a detecção de casos de infecção ativa. Sua sensibilidade é superior a $90 \% \mathrm{em}$ pacientes sintomáticos ou não e o seu valor preditivo negativo chega a $100 \%$, de forma que o risco para o desenvolvimento de doença por CMV é zero na ausência de antigenemia positiva. ${ }^{21}$

Portanto, o grande desafio é definir o que é mais seguro na prática clínica: a profilaxia universal ou a terapia preemptiva baseada em testes seriados de antigenemia. Somente estudos randomizados ou comparativos utilizando um grupo controle contemporâneo ou histórico poderá nos esclarecer e, com isso, auxiliar na decisão pela melhor conduta em nosso centro.

\section{CONCLUSÃO}

A incidência de infecção ativa por CMV em transplante de pulmão foi elevada $(68 \%)$ a despeito do regime de profilaxia com GIV nos primeiros três meses. Embora a intensidade da infecção seja geralmente leve, a utilização da profilaxia universal é discutível quando da disponibilidade de testes diagnósticos tão sensíveis quanto a antigenemia. A relação entre a ocorrência de infecção ativa por CMV e mortalidade precoce e tardia deve ser melhor estudada, devendo-se considerar as causas dos óbitos ocorridos. 


\begin{abstract}
Objective: Determine cytomegalovirus infection incidence in lung transplantation under universal intravenous ganciclovir prophylaxis, and analyzing its effect on the lung transplant survival. Methods: 106 lung transplants performed between March/99 and Feb/04, 82 were studied, 24 were excluded due to death in the first month $(\mathrm{n}=24 ; 22.6 \%)$. Recipients received cyclosporine, azatioprine, and prednisone. Prophylactic ganciclovir was administered $10 \mathrm{mg} / \mathrm{kg} /$ day for 3 weeks, followed by $5 \mathrm{mg} / \mathrm{kg} / \mathrm{day}$, and 3x/week between 4th and 12 th weeks. Whenever active infection was present, patients received ganciclovir $10 \mathrm{mg} / \mathrm{kg} / \mathrm{day}$ until attaining two negative antigenemia or after finishing the 21 days treatment. Peripheral blood pp65 cytomegalovirus antigenemia was used to identify active infection between weeks 3rd to 12th. It was monthly used from $4^{\text {th }}$ to $12^{\text {th }}$ month or whenever presenting clinical suspicion. Active infection was defined as any positive cell among 105 granulocytes. It was employed t Student, Ki-square and Fisher's Exact tests, mean \pm standard deviation, Kaplan Meier; it was considered significant $p<0,05$. Results: The incidence of cytomegalovirus infection was $68.3 \%(56 / 82)$. Maximum median antigenemia was 11 cells (1-1096). Median time to attain positive antigenemia was 114 days (28-343). There was no difference concerning the gender distribution $(\mathrm{p}=0.8)$, age $(\mathrm{p}=0.599)$, and type of donor $(\mathrm{p}=0.375)$ between patients with and without cytomegalovirus infection. Death rate during follow-up period was of $40.2 \%(33 / 82), 19.5 \%(16 / 82)$ in the first year without difference between groups. Conclusion: It was attained a high CMV active infection incidence among lung transplant recipients despite the intravenous ganciclovir universal prophylaxis.
\end{abstract}

Key words: cytomegalovirus, antigenemia, lung transplant, ganciclovir.

\section{REFERÊNCIAS}

1. Drago F, Aragone MG, Lugani C, Rebora A. Cytomegalovirus infection in normal and immunocompromised humans. A review. Dermatology 2000;200(3):189-95.

2. Abbas A, Lichtman A, Jordan P. Cellular and Molecular Immunology. In:. 4 ed; 2002. p. 4-14.

3. de Jong MD, Galasso GJ, Gazzard B, Griffiths PD, Jabs DA, Kern ER, et al. Summary of the II International Symposium on Cytomegalovirus. Antiviral Res 1998;39(3):141-62.

4. Schroeder R, Mesko J, Santos A, Keitel E, Bittar A, Garcia V, et al. Cytomegalovirus antigenemia and renal function post-kidney-transplantation. Transplant Proc 1999;31(7):3027-8.

5. Allen CT, Bag R, Zander DS, Cagle PT. Cytomegalovirus infection masquerading as carcinoma in a lung transplant patient. Arch Pathol Lab Med 2005;129:e1.

6. Westall PG, Michaelides A, Williams JT, Snell IG, Kotsimbos CT. Bronchiolitis obliterans syndrome and early human cytomegalovirus DNAaemia dynamics after lung transplantation. Transplantation 2003;75(12):2064-2068.

7. Danzinger-Isakov LA, DelaMorena M, Hayashi RJ, Sweet S, MendeloffE, Schootman $\mathrm{M}$, et al.Cytomegalovirus viremia associated with death or retransplantation in pediatric lung-transplant recipients. Transplantation 2003;75(9):1538-1543.

8. Brumble LM, Milstone AP, Loyd JE, Ely EW, Pierson RN, Gautam S, Dummer JS. Prevention of cytomegalovirus infection and disease after lung transplantation: Results using a unique regimen employing delayed ganciclovir. Chest 2002;121(2)407.

9. Limaye AP, Raghu G, Koelle DM, Ferrenberg J, Huang M, Boeckh M. High incidence of ganciclovir-resistant cytomegalovirus infection among lung transplant recipients receiving preemptive therapy. The Journal of Infectious Disease 2002;185:20 20-27.

10. Zamora MR. Controversies in lung transplantation: Management of cytomegalovirus infection.The Journal of Heart and Lung Transplantation 2002;21(8):841-849.

11. Schnitzler. The effects of Cytomegalovirus. Am J Kidney Dis 1999.

12. Schroeder R, Michelon T, Fagundes I, Bortolotto A, Lammerhirt E, Oliveira J et al.
Cytomegalovirus disease latent and active infection rates during the first trimester after kidney transplantation . Transplantation Proceedings 2004;36:896-898.

13. Valentine HA, Luikart H, Doyle R, Theodore J, Hunt S, Oyer P et al. Impact of cytomegalovirus hyperimmune globulin on outcome after cardiothoracic transplantation. Transplantation 2001;72(10)1647-1652.

14. Kruger RM, Paranjothi S, Storch GA, Lynch JP, Trulock EP. Impact of prophylaxis with CytoGan alone on the incidence of CMV viremia in CMV seropositive lung transplant recipients. Journal of Heart and Lung Transplantation 2002;22(7):754-763.

15. Zamorra MR. Cytomegalovirus and lung Transplantation. American Journal of Transplantation 2004;4:1219-1226.

16. Danzinger-Isakov LA, Faro A, Sweet S, Michaels MG, Aurora P, Mogayzel PJ et al. Variability in standard care for cytomegalovirus prevention and detection in pediatric lung transplantation: Surveyor eight pediatric lung transplant programs. Pediatr Transplantation 2003;7:469-473.

17. Palmer SM, Grinnan DC, Reams BD, Steele MP, Messier RH, Davis RD. Delay of CMV infection in high-risk CMV mismatch lung transplant recipients due to prophylaxis with oral ganciclovir. Clin Transplant 2004;18:179-185.

18. WeilL D, Lock BJ, Wewers DL, Young KR, Zorn GL, Early L et al. Combination prophylaxis with ganciclovir and cytomegalovirus (CMV) immune globulin after lung transplantation: Effective CMV prevention following daclizumab induction. American Journal of Transplantation 2003;3:492-496.

19. Perreas KG, McNeil K, Charman S, Sharples LD, Wreghitt T, Wallwork J. Extended ganciclovir prophylaxis in lung transplantation. Journal of Heart and Lung Transplantation 2005;24(5):585-587.

20. Bhorade SM, Lurain NS, Jordan A, Leischner J, Villanueva J, Durazo R et al Emergence of ganciclovir-resistant cytomegalovirus in lung transplant recipients. Journal of Heart and Lung Transplantation 2002;21(12):1274-1282.

21. Schroeder R, Michelon T, Fagundes I, Bortolotto A, Lammerhirt E, Oliveira J et al. Antigenemia for cytomegalovirus in post renal transplantation: Choosing a cut off for the diagnosis criteria in cytomegalovirus disease. Transplantation Proceedings 2005; 37(6): 2781-2783. 